Rapid Reviews COVID-19

\title{
Review 2: "Deaths of Despair and the Incidence of Excess Mortality in 2020"
}

\section{Rudi Rocha ${ }^{1}$}

${ }^{1}$ FGV-EAESP: Fundacao Getulio Vargas Escola de Administracao de Empresas de Sao Paulo, Economics, Brazil

Published on: Feb 22, 2021

License: Creative Commons Attribution 4.0 International License (CC-BY 4.0). 


\section{$\underline{\text { RR:C19 Evidence Scale rating by reviewer: }}$}

- Reliable. The main study claims are generally justified by its methods and data. The results and conclusions are likely to be similar to the hypothetical ideal study. There are some minor caveats or limitations, but they would/do not change the major claims of the study. The study provides sufficient strength of evidence on its own that its main claims should be considered actionable, with some room for future revision.

\section{Review:}

This paper examines whether the COVID-19 pandemic is associated with an increase in deaths of despair in the US. The author relies on the MCOD 2015-2018 files to project 2020 deaths by age groups and sex through early October. Projected series are then compared to actual deaths from CDC files for 2020, including those by COVID-19. Excess deaths are defined as the difference between actual and projected deaths, and non-COVID excess mortality is defined as the difference between excess deaths and COVID-19 deaths. Estimates suggest that mortality in 2020 exceeds what would have occurred if official COVID-19 deaths were combined with the expected number of deaths from other causes. The author then compares previous patterns of deaths of despair (by age, sex, and season) with patterns of the non-COVID excess deaths. That mapping suggests that the pandemic is associated with an increase in deaths of despair (10 to 60 percent) above already high pre-pandemic levels.

This is an extremely relevant topic and the paper delivers a strong and timely message. However, to what extent is the phenomenon related to substance abuse and the US opioid epidemic specifically? To what extent is the phenomenon related to a harvest effect? There is increasingly dense and relevant literature connecting COVID-19 and mental health issues worldwide, including concerns directly related to suicide risk in many developed countries. I would be interested to read the author's thoughts on the question of whether the increase in excess of deaths in general, and in deaths of despair in particular, is differentially salient in the US and go beyond more typical effects on mental health that one would expect in peer countries.

On the empirical front, the claims of the paper are generally reliable and trustworthy. Results are grounded on convincing empirical and descriptive methods. The author conducts a very straightforward analysis, identifies excess deaths in general, and provides correlational evidence supporting the view that part of the excess deaths is likely connected to an increase in deaths of despair. I suggest two additional simple exercises to give further support to the narrative. First, cells could be split into smaller ones (e.g. age groups into 5-year brackets) so that to improve the sample 
size and the descriptive analysis shown in Figure 4. Second, as a final falsification test, the author could replace the variable in the $\mathrm{x}$-axis in Figure 4 with the change in deaths of despair between 2017 and 2018. If trends were indeed well estimated, we should not observe any association between y and delta $\mathrm{x}$.

Finally, there is dense literature on the relationship between the COVID-19 pandemic and mental health issues. Some recent developments and insights on the topic could be considered. This should be important in the discussion on whether the US is an outlier or not, and on the extent to which the existing opioid epidemic has indeed leveraged the number of deaths much beyond expected figures. The literature has also been rich in the discussion on how countries should deal with or contain suicide risks and substance abuse amidst the COVID-19 pandemic. I recommend the reading of Gunnell et al. (June 2020), published in The Lancet Psychiatry v7(6), p468-471. 